\title{
Novel cis-element in intron 1 represses somite expression of zebrafish myf-5
}

\author{
Cheng-Yung Lin, Yau-Hung Chen, Hung-Chieh Lee, Huai-Jen Tsai* \\ Institute of Molecular and Cellular Biology, National Taiwan University, Room 307A, Fisheries Science Building, \\ 1 Roosevelt Road, Section 4, Taipei 106, Taiwan
}

Received 6 October 2003; received in revised form 17 February 2004; accepted 9 March 2004

Available online 12 May 2004

Received by R. di Lauro

\begin{abstract}
Myf-5 is a basic helix-loop-helix (bHLH) transcription factor that controls muscle differentiation. During early embryogenesis, myf-5 expression is transient, somite- and stage-specific. However, the negative regulation of myf-5 is poorly understood. We constructed a plasmid $[(-9977 /-1) / \mathrm{E} 1 / \mathrm{I} 1 / \mathrm{E} 2 / \mathrm{GFP}]$ that contains the sequence -9977 to -1 , exon 1 (E1), intron 1 (I1), and exon 2 (E2) of zebrafish (Danio rerio) $m y f-5$ and a reporter GFP gene. This plasmid was microinjected into zebrafish zygotes. Surprisingly, the somite-specific expression rate of reporter GFP in the transgenic embryos was extremely low $(2 \%, n=392)$, compared to that of $(-9977 /-1) / \mathrm{GFP}(92 \%, n=210)$. Dramatic repression of $m y f-5$ expression was also observed in embryos microinjected with plasmids in which the sequence $-8600 /-1$, $-2937 /-1$ or $-290 /-1$ was linked to E1/I1/E2/GFP. Thus, intron 1 contains a silencer that specifically represses the activity of $m y f-5$. Functional analysis of intron 1 showed a strong, negative, cis-regulatory element was located at $+502 /+835$. Its function was orientationand position-dependent. The repressive capability of this silencer was completely dependent on two core motifs, IE1 ( + 502/ +527) and IE2 $(+816 /+835)$, and a 156-bp spanning sequence that lies between them. This is the first study to identify a novel, cis-acting silencer in intron 1 that is crucial to negatively regulating zebrafish myf-5 expression.
\end{abstract}

(C) 2004 Elsevier B.V. All rights reserved.

Keywords: Myf-5; Intron; Somite; Regulatory cis-elements; Repression

\section{Introduction}

Myogenic regulatory factors (MRF) are transcription factors in the basic-helix-loop-helix (bHLH) family. They are essential for skeletal muscle determination and differentiation (Edmondson and Olson, 1993). They activate musclespecific transcription by binding to a DNA consensus sequence, an E-box, present in the promoter of numerous muscle-specific genes (Smith et al., 1994). Each bHLH MRF may play a different role during myogenesis. Primary MRF,

Abbreviations: BAC, bacterial artificial choromosome; bHLH, basichelix-loop-helix; bp, base pair(s); cDNA, DNA complementary to RNA; dpc, days post coitum; GFP, green flourecence protein; hpf, hours postfertilization; IE, intron element; kb, kilobase(s); MRF, myogenic regulatory factors; PCR, polymerase chain reaction; RT, reverse transcriptase.

* Corresponding author. Tel.: +886-2-2364-1705; fax: +886-2-23638483.

E-mail address: hjtsai@ntu.edu.tw (H.-J. Tsai).
MyoD and Myf-5, are involved in myoblast specification, while secondary MRF, Mygenin and MRF4, are involved in myotube formation (Yablonka-Reuveni and Rivera, 1994; Rudnicki and Jaenisch, 1995; Cornelison and Wold, 1997).

The expression pattern of $m y f-5$ reveals its role during the early determination of the myogenic lineage. $m y f-5$ is the first MRF to be expressed in mammals (reviewed by Buckingham, 1992), birds (Hacker and Guthrie, 1998; Hirsinger et al., 2001) and fish (Chen et al., 2001). In mouse embryos, myf-5 expression begins in the trunk 8 days post coitum (dpc), but disappears $14 \mathrm{dpc}$. In Xenopus, myf-5 is first activated in the dorsal region of stage 10 embryos (Jones and Smith, 1998; Takahashi et al., 1998). Soon after the onset of gastrulation, the expression domain of Xenopus myf-5 shifts to the dorsolateral marginal zone. This zone may give rise to the anterior somatic mesoderm. As gastrulation proceeds, myf-5 expression in the future anterior somatic mesoderm is progressively down-regulated and $m y f-5$ transcripts are found only in the 
posterior part of the somatic mesoderm (Dosch et al., 1997). In zebrafish, myf-5 transcripts are detectable at 7.5 hours post-fertilization (hpf), primarily in the somites and segmental plates. Prominent, transient signals of $m y f-5$ occur in adaxial cells, in two parallel rows, but they do not extend beyond the positive-signal somites (Chen et al., 2001; Coutelle et al., 2001). myf-5 transcripts increase substantially until $16 \mathrm{hpf}$, and then gradually decline to undetectable levels by $26 \mathrm{hpf}$ (Chen et al., 2001). During embryogenesis, myf-5 expression is transient, somite-specific and stage-dependent. Thus, $m y f-5$ is strongly repressed after somitogenesis by an unknown mechanism.

Activation of $m y f-5$ is controlled by lineage-specific transcription enhancers, suggesting that complicated mechanisms control progenitor specification at different myogenic sites in the embryo. Promoter analysis of mouse myf-5 focusing on the long-range, or distal, fragment of modulation demonstrates that all essential, upstream myf-5 regulatory sequences occur within $-96 \mathrm{~kb}$ (Hadchouel et al., 2000). Bacterial artificial choromosome (BAC) transgenic analysis of the myf-5 locus reveals that complex elements control activation and maintenance of gene expression during muscle development (Carvajal et al., 2001). Several cis-elements have been proposed, including the distal regulatory element ( $-96 /-63 \mathrm{~kb})$, proximal promoter $(-58 /-48 \mathrm{~kb})$, central nervous system-specific enhancer $(-0.5 /-0.1 \mathrm{~kb})$, and the hypaxial $(+0.5 /+3.5 \mathrm{~kb})$, epaxial $(-5.6 /-4.6 \mathrm{~kb})$, and branchial myotomes $(-1.5 /-0.5$ and $+0.5 /+3.5 \mathrm{~kb})$ (Summerbell et al., 2000). In Xenopus, the T-box binding site is responsible for initiating $m y f-5$ expression in the dorsal mesoderm of embryos (Lin et al., 2003). In zebrafish, microinjection of various upstream fragments in the F0 generation demonstrates that some somite-specific elements exist within $-6.2 \mathrm{~kb}(-6216 /-1)$. The $-82 /-1$ region is the minimal element required for somite-specific expression of zebrafish myf-5 (Chen et al., 2001) and the proximal regulatory cis-element $-82 /-62$ cassette drove somitespecific expression and repressed nonspecific expression during the early development of zebrafish embryos (Chen et al., 2003). In Xenopus, a TCF-3 binding site prevents $m y f-5$ expression in dorsal midline mesoderm (Yang et al., 2002) and a proximal, interferon regulatory factor binding element is required for the down-regulation of $m y f-5$ expression in differentiating myocytes (Mei et al., 2001). However, the mechanism by which zebrafish myf-5 expression is repressed is completely unknown.

In this study, in vivo transgenesis was used to study the functional roles of intron 1 of zebrafish myf-5. We found two cis-acting elements in intron 1, IE1 and IE2, that function together to repress $m y f-5$ transcription in zebrafish embryos. Furthermore, for IE1 and IE2 to function correctly, we determined that they must be separated by $156 \mathrm{bp}$, whose sequence in unimportant. This suggests that chromatin remodeling may help maintain static repression of $m y f-5$. This is the first study to show intron 1 has an important role in repressing $m y f-5$ expression.

\section{Materials and methods}

\subsection{Experimental fish}

Zebrafish (AB strain) were reared at approximately $28.5{ }^{\circ} \mathrm{C}$, under a $14 \mathrm{~h} \mathrm{light} / 10 \mathrm{~h}$ dark photoperiod. After fertilization, eggs were collected and cultured in an aquarium. Embryonic cleavage number and somite formation were observed with a light microscope to determine the developmental stage (Kimmel et al., 1995).

\subsection{Plasmid constructions}

All oligonucleotide sequences used in this study are shown in Table 1. For promoter deletion experiments, primers G0.3-EcoRIF and G0.3-AgeIR were used to amplify a $2.9-\mathrm{kb}$ polymerase chain reaction (PCR) product from a template of recombinant phage DNA (Chen et al., 2001). The PCR product contained the $0.3-\mathrm{kb}$ sequence upstream $(-0.3 \mathrm{~kb})$ of $m y f-5,0.5-\mathrm{kb}$ exon $1,2-\mathrm{kb}$ intron 1 and $0.1-\mathrm{kb}$ exon 2. The PCR product was inserted into pGEM-T Easy (Promega) vector to produce a plasmid (pG0.3). The 2.9-kb fragment of EcoRI-AgeI-digested pG0.3 was ligated to EcoRI-AgeI-digested pEGFP-1 (Clontech) vector to generate $\mathrm{pPG} 0.3$, in which GFP was fused in frame. A fragment obtained from the EcoRI-PstI-digested phage DNA was ligated to the EcoRI-PstI-digested pPG0.3 vector to generate pPG8.6. The EcoRV-NotI-digested pPG8.6 was ligated to EcoRV-NotI-digested pP10.0 (myf-5 positive phage DNA used SacI-digested and the fragment was ligated to pZMYP-6212E; Chen et al., 2001) and pP3.0 (pZMYP2937E, Chen et al., 2001) vectors to generate pPG10.0 and pPG3.0, respectively.

A variety of intron I deletions were constructed with PCR products generated from the template of the recombination phage. Primer pairs F1/R8, F5/R8, F7/R8, F4/R5, F6/R7, F1/R6, F1/R4, F1/R3, F1/R1, F1/R2, F3/R3, F2/R3 and F2/ $\mathrm{R} 2$ were used to produce $2,1.4,0.7,0.7,0.7,1.3,0.7,0.4$, $0.2,0.3,0.3,0.3$ and $0.3 \mathrm{~kb}$ of PCR product, respectively. The PCR products were ligated into pGEM-T Easy vector. The resulting plasmids were digested with NotI and ligated to the NotI-digested pP3.0 to generate pP3.0 I 1-I 8 and pP3.0 I 10-I 14.

To determine the function of the $+660 /+815$ fragment within $m y f-5$ intron 1, this fragment was cut from pP3.0 I 8 with $X b a \mathrm{I}$ and HindIII. Then, blunting and self-ligation were used to generate plasmid pP3.0 I 8 D. For PCR reactions, $m y f-5$ intron 1 DNA was used as a template for derivate plasmids pP3.0 I 8 D1-D4, and pGEM-T Easy vector was used as a template for pP3.0 I 8 D5-D6. Primer sets $\mathrm{d} 1 \mathrm{~F} / \mathrm{d} 1 \mathrm{R}, \mathrm{d} 2 \mathrm{~F} / \mathrm{d} 2 \mathrm{R}, \mathrm{d} 3 \mathrm{~F} / \mathrm{d} 3 \mathrm{R}, \mathrm{d} 4 \mathrm{~F} / \mathrm{d} 5 \mathrm{R}, \mathrm{d} 5 \mathrm{~F} / \mathrm{d} 5 \mathrm{R}$ and $\mathrm{d} 6 \mathrm{~F} / \mathrm{d} 6 \mathrm{R}$ were used to produce $51,44,45,33,156$ and $156 \mathrm{bp}$ PCR products, respectively. The PCR products were ligated into pGEM-T Easy vector. All plasmids were digested with $X b a \mathrm{I}-H i n \mathrm{dIII}$ and then ligated to the $X b a \mathrm{I}-H i n d I I I-d i g e s t e d$ pP3.0 I 8 vector to generate pP3.0 I 8 D1-D6. 
To understand the function of the $m y f-5$ intron 1 fragment, plasmid pP3.0 was digested with NotI, blunted and fused with an irrelevant $2-\mathrm{kb}$ fragment recovered from HindIII-cut $\lambda$ phage. Plasmids with two directions of $2-\mathrm{kb}$ fragment were generated, pP3.0 $22 \mathrm{~K}(+)$ and pP3.0 $22 \mathrm{~K}(-)$. The plasmid pCMVm, which has CMV promoter fused with GFP (Wang et al., 2002b), and intron $1(+502 /+2503)$ digested with NotI from pGEM-T Easy vector, $\mathrm{p} I$ 1, were ligated to the NotI-digested pCMVm to produce plasmids pCMV I $1(+)$ and pCMV I $1(-)$, which contain both directions of insert.

\subsection{Reverse transcriptase-PCR (RT-PCR)}

Embryos that had developed for $24 \mathrm{hpf}$ were pooled and their RNA was extracted as described by Chen et al. (2001). However, primer 1 and primer 2 were used for PCR, with denaturation for $30 \mathrm{~s}$ at $94{ }^{\circ} \mathrm{C}$ and annealing for 1 min at $55^{\circ} \mathrm{C}$.

\subsection{Microinjection, green fluorescence detection and} whole-mount in situ hybridization

The procedures for microinjection and whole-mount in situ hybridization were described by Chen et al. (2001). The methods and equipments used for green fluorescence detection were also described by Chen et al. (2001). However, we observed GFP expression of transgenic embryos hourly, especially from 6 to $36 \mathrm{hpf}$. The GFP expression rate and the somite expression rate were calculated as described in Chen et al. (2001). At $30 \mathrm{hpf}$, we counted the number of embryonic muscle fibers exhibiting a GFP-positive signal. From 1 to 10 GFP-positive fibers was categorized as a low level of expression. From 11 to 40 GFPpositive fibers was medium and from 41 to 70 GFP-positive fibers was categorized as a high level of expression. The percentage of embryos exhibiting each level of expression was calculated.

\section{Results}

3.1. Downstream cis-element affects zebrafish myf-5 gene expression

To understand the molecular mechanism that controls the somite- and stage-specificity of $m y f-5$, we obtained a -10 $\mathrm{kb}$ fragment from recombinant phage DNA (Chen et al., 2001). We generated plasmid pP10.0, which was comprised of the fragment from -9977 to -1 of zebrafish $m y f-5$ fused

Table 1

Primer sequences $\left(5^{\prime} \rightarrow 3^{\prime}\right)$

\begin{tabular}{|c|c|}
\hline G0.3-SacIF & CGCGAGCTCTCAAAGTCTACATCTAAA \\
\hline G0.3-AgeIR & GGTGGCGACCGGTACAGGACTGTTGCA \\
\hline $\mathrm{F} 1$ & ATGGTAAATATGACTCTCTTTCTCT \\
\hline $\mathrm{F} 2$ & TCTATGGGGTTTAAACATGATAGAC \\
\hline F3 & CTACAGTGAAGCTCTGCATAAACTC \\
\hline F4 & GGCTCCTCCTTTTACGCTTAGATA \\
\hline F5 & TGTTCATTCACTCATTTTCTTTTCG \\
\hline F6 & TTAAATGACTACAATACTGTTGG \\
\hline F7 & GACTCTAAAAAATGCTGGAT \\
\hline $\mathrm{R} 1$ & GACACAATGAAGAAGAGAGAAACCT \\
\hline $\mathrm{R} 2$ & TATATATCTAAGCGTAAAAG \\
\hline R3 & TGGACAAACAGCAGTAAGC \\
\hline R4 & CGAAAAGAAAATGAGTGAATGAACA \\
\hline R5 & CCAACAGTATTGTAGTCATTTAA \\
\hline R6 & ATCCAGCATTTTTTAGAGTC \\
\hline R7 & GATTTACTGAGGCTTTATTGGGC \\
\hline $\mathrm{R} 8$ & GCAGTCAACCTACAATGACAAA \\
\hline $\mathrm{d} 1 \mathrm{~F}$ & CTAGACATGGCAATTGTACTGTGTTTGGTCTTTATATTTTATCTCAATAG \\
\hline $\mathrm{d} 2 \mathrm{~F}$ & CTAGAAATAGCACACGGGGGATTGTATCCTGCAAATGGAGTCAGAGCTGCA \\
\hline $\mathrm{d} 3 \mathrm{~F}$ & CTAGAGCTGCCTCAGTCTCAGCATGACAACAATAAATTGCTGTCTACATCA \\
\hline $\mathrm{d} 4 \mathrm{~F}$ & CTAGAACATCTGGCTCCTCTTTTACGCTTAGATATATA \\
\hline $\mathrm{d} 5 \mathrm{~F}$ & TCTAGATAATACGGTTATCCACAG \\
\hline $\mathrm{d} 6 \mathrm{~F}$ & TCTAGATGAGCGTCGATTTTTGTGAT \\
\hline $\mathrm{d} 1 \mathrm{R}$ & AGCTTCTATTGAGATAAAATATAAAGACCAAACACAGTACAATTGCCATGT \\
\hline $\mathrm{d} 2 \mathrm{R}$ & AGCTTGCAGCTCTGACTCCATTTGCAGGATACAATCCCCCGTGTGCTATTT \\
\hline $\mathrm{d} 3 \mathrm{R}$ & AGCTTGATGTAGACAGCAATTTATTGTTGTCATGCTGAGACTGAGGCAGCT \\
\hline d4R & AGCTTATATATCTAAGCGTAAAAGAGGAGCCAGATGTT \\
\hline $\mathrm{d} 5 \mathrm{R}$ & AAGCTTTGAGCGTCGATTTTTGTG \\
\hline d6R & AAGCTTTAATACGGTTATCCACAGAA \\
\hline Primer 1 & TCAGGAGCTCCTCAGGGAACAAGTG \\
\hline Primer 2 & ACTTGTACAGCTCGTCCATGCCGA \\
\hline
\end{tabular}


with GFP gene (Fig. 1). Transient expression assays in F0 embryos showed that GFP was first detectable at $5.25 \mathrm{hpf}$. This was accordant with the initial expression pattern of endogenous myf-5, which was determined using wholemount in situ hybridization (Fig. 1). Strong GFP signals were apparent in muscle fibers at $30 \mathrm{hpf}$ (Fig. 1I), and were detected continuously after $96 \mathrm{hpf}$ (data not shown). Plasmids pP8.6, pP3.0 and pP0.3, which contained different lengths of the upstream region, also were constructed (Fig. 1). Four counterpart plasmids (pPG10.0, pPG8.6, pPG3.0 and $\mathrm{pPG} 0.3$ ), in which exon 1 , intron 1 , and part of exon 2 were fused, were generated. The GFP expression rate in the muscle fibers and somites of embryos injected with plasmid pP10.0, pP8.6, pP3.0 or pP0.3 ranged from $85 \%$ to $96 \%$. In contrast, the GFP expression rate was only $2-5 \%$ in embryos injected with plasmid pPG10.0, pPG8.6, pPG3.0 or pPG0.3 (Fig. 1J-M). RT-PCR was used to detect mRNA transcripts in the injected embryos. An 841-bp PCR product was generated, indicating that the boundaries between exon 1 and intron 1, and intron 1 and exon 2 were spliced correctly (Fig. 2). Therefore, we conclude that the downstream sequence of zebrafish $m y f-5$ controls repression of the gene.
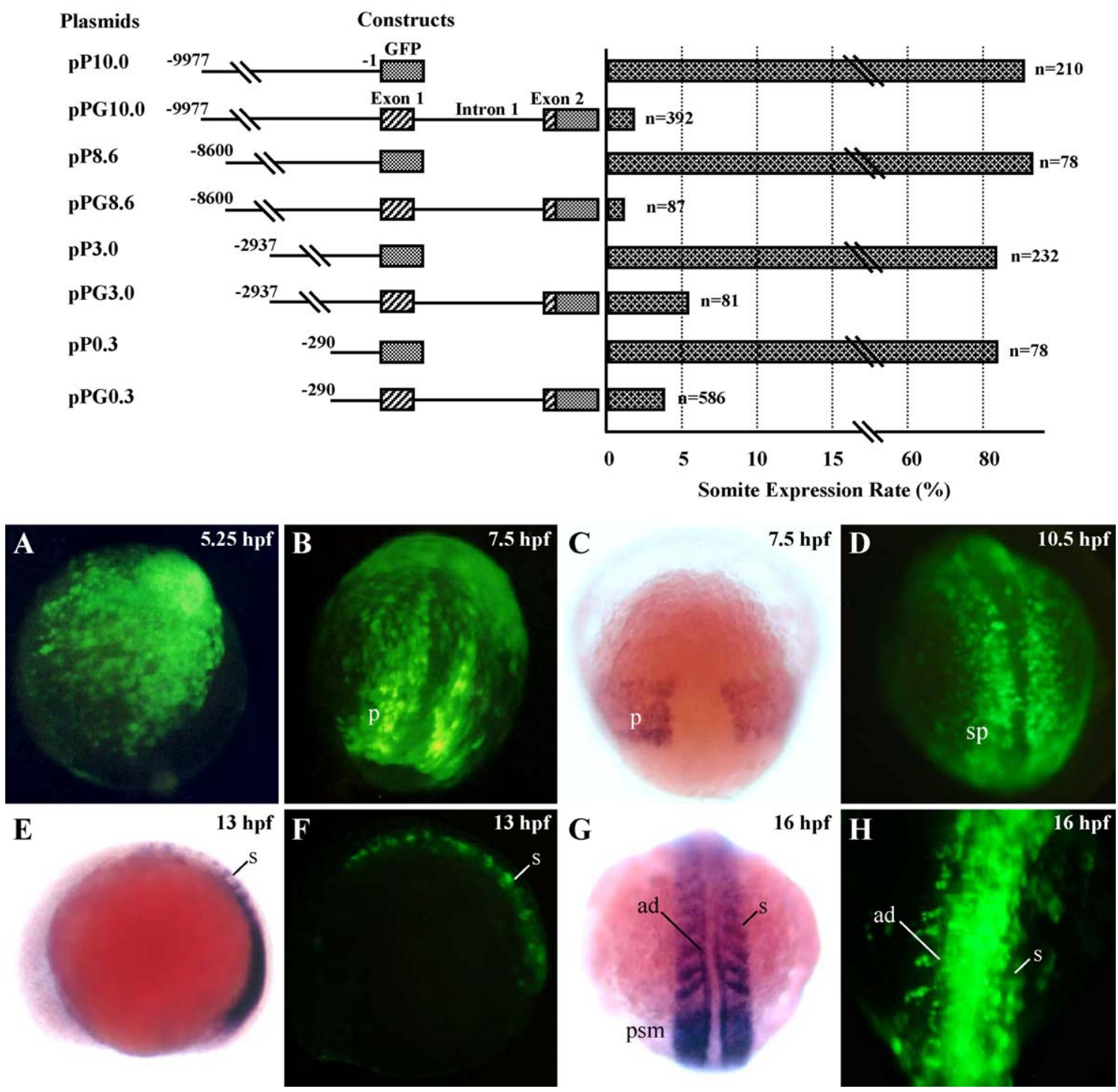

Fig. 1. A downstream cis-element is involved in the repression of zebrafish $m y f-5$. Plasmids used for the transient GFP assay were microinjected into the 1-cell stage of fertilized eggs. $n$ : the total number of live embryos examined; somite expression rate: the number of embryos that expressed GFP in the somites divided by the total number of embryos examined. Dynamic expression patterns of GFP in the F0 embryos injected with $-9977 /-1 /$ GFP of zebrafish myf-5 (pP10.0). Strong GFP signals were first detected $5.25 \mathrm{hpf}$ (A). The signals extended to two triangular regions $7.5 \mathrm{hpf}$ (B), to the segmental plate $10.5 \mathrm{hpf}$ (D), and to the somites $13(\mathrm{~F})$, and $16 \mathrm{hpf}(\mathrm{H})$. The GFP patterns in the $\mathrm{pP} 10.0$-injected $\mathrm{F} 0$ embryos were able to recapitulate the endogenous transcription patters of $m y f-5$ at 7.5- (C), 13- (E), and 16-hpf (G) embryos by whole mount in situ hybridization using myf-5 riboprobe. p: paraxial mesoderm segmental plate, sp: segmental plate, s: somite, ad: adaxial cells, psm: presomitic mesoderm. The GFP expression patterns in 30-hpf transgenic embryos injected with each construct, as indicated, were photographed (exposure: $8 \mathrm{~s}$ ) with a fluorescence microscope. Arrows indicate GFP expression in muscle fibers. WT: wild-type untreated embryo. Scale bars: $250 \mu \mathrm{m}$. 

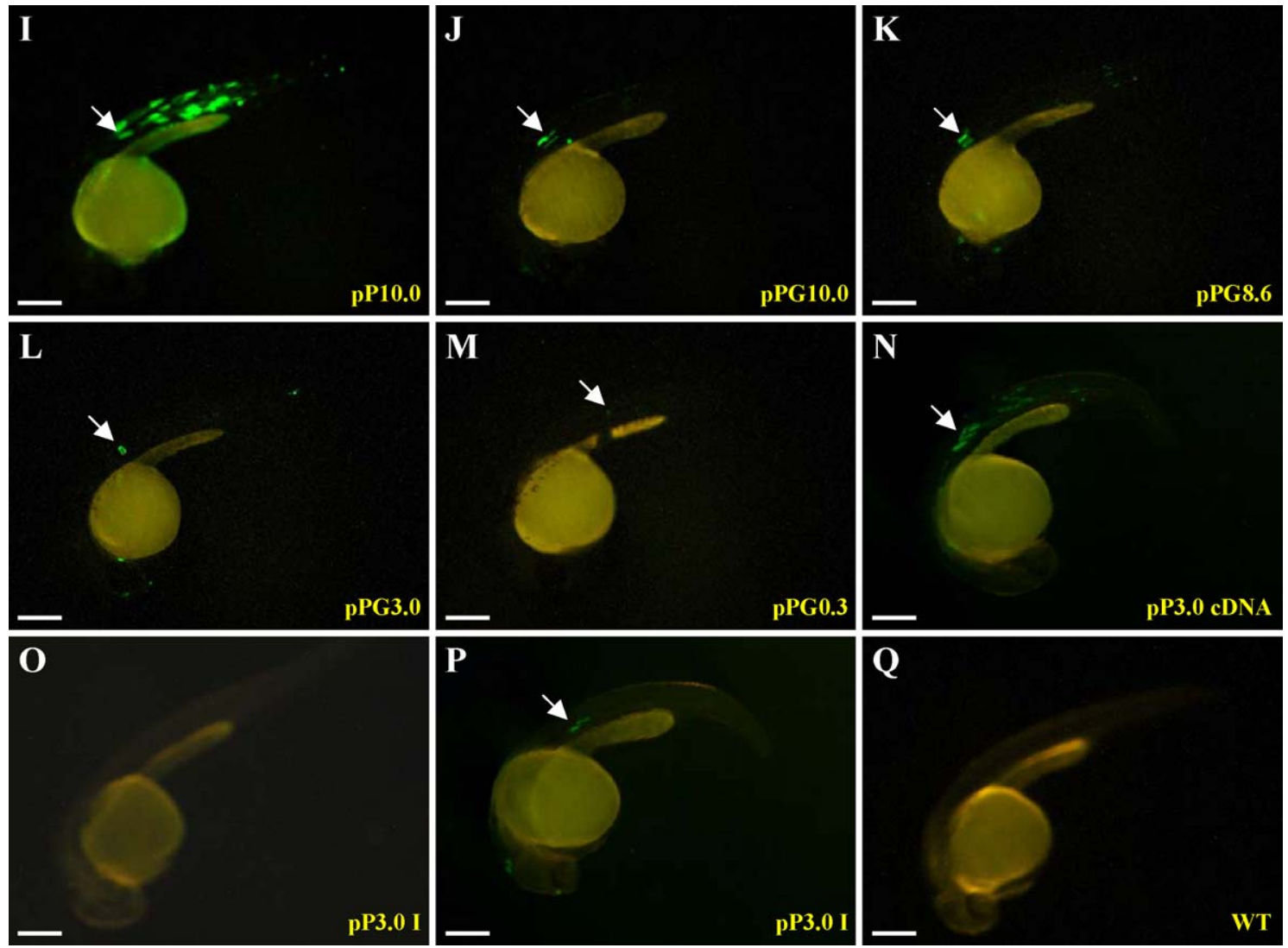

Fig. 1 (continued)

\subsection{Intron 1 of myf-5 controls gene repression}

We constructed two plasmids to identify the element(s) in the downstream region of $m y f-5$ (including exon 1, intron 1 and exon 2) that mediate gene repression. Plasmid pP3.0 cDNA contained the fragment $-2937 /-1$, myf-5 cDNA and the GFP gene. Plasmid pP3.0 I 1 contained the fragment $-2937 /-1$, the GFP gene and intron 1. GFP was expressed in the muscle fibers of somites of embryos injected with plasmid pP3.0 cDNA (Fig. 1N). However, GFP signals were not detected or very weak in embryos injected with pP3.0 I 1 (Fig. $1 \mathrm{O}$ and P), which contained intron 1 of $m y f-5$. Thus, the repressive element is located in intron 1 . Several additional plasmids were constructed to determine the smallest segment of intron 1 that was capable of repressing $m y f-5$. Repression of GFP expression decreased greatly in transgenic embryos injected with pP3.0 I 1, I 2, I 3, I 4 or I 5 (Fig. 3). However, repression was restored in transgenic embryos injected with pP3.0 I 6, I 7 or I 8, which contained the segments $+502 /$ $+1787,+502 /+1199$ and $+502 /+835$, respectively. In addition, there was no repression in embryos injected with either pP3.0 I 8 D or pP3.0 I 10 (Fig. 3). In these plasmids, $+660 /+815$ was missing from the $+502 /+835$ fragment. The rate of GFP expression in transgenic embryos injected with either pP3.0 I 8 D or pP3.0 I 10 was around 10-fold that of embryos injected with pP3.0 I 8, indicating that +660 / +815 is the key segment in the repression of myf -5 .
3.3. myf-5 repressive element is orientation- and positiondependent

Full-length intron I $(+502 /+2503)$ or the repressive fragment $(+502 /+835)$ was linked to myf5/GFP in both directions. Transient GFP assays demonstrated that both intron 1 and the repressive element, when on the sense strand, repressed GFP expression (Fig. 4). However, neither repressed GFP expression when located on the antisense strand. Similar results were obtained when an irrelevant DNA fragment, such as lambda phage $22 \mathrm{~K}$, was microinjected (Fig. 4). Furthermore, when the repressive element was positioned in the front of $-2937(\mathrm{pP}$ 3.0 I 9), repression was nearly abolished (Fig. 4). GFP, driven by a CMV promoter with the full length of intron 1 ligated downstream in two directions, was expressed in all cell types, not only muscle. In this position, the intron 1 fragment lost its repressive efficacy. This evidence clearly demonstrates that inactivation of $m y f-5$ by the repressive element within intron 1 is orientation- and position-dependent.

\subsection{A nucleotide spacer is required for repression}

Repression was nearly lost when $+660 /+815$ was removed from $+502 /+835$ fragment (Fig. 3). To analyze the $+660 /+815$ motif in more detail, we constructed four 

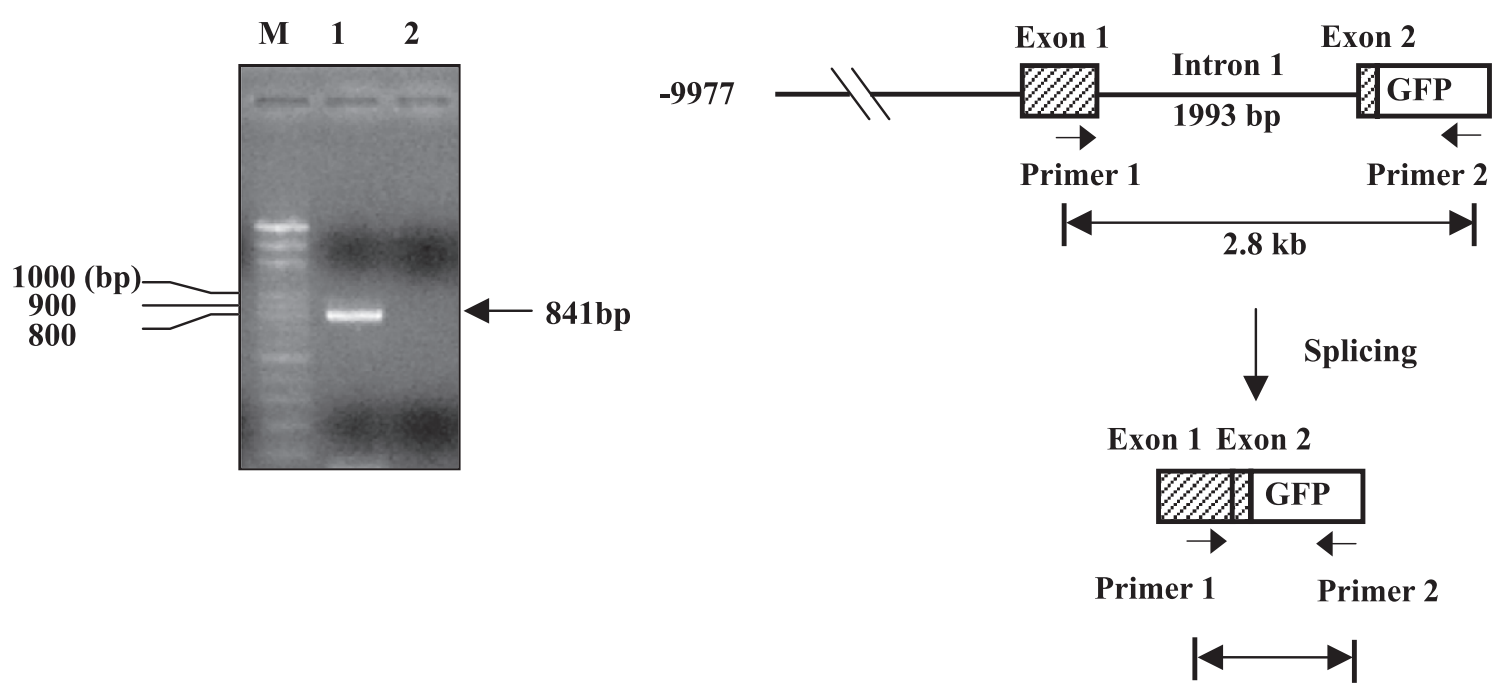

841 bp

Fig. 2. Processing of $m y f-5$ transcripts was detected with RT-PCR. A forward primer 1 , corresponding to the myf-5 exon 1 , and a reverse primer 2 , corresponding to the GFP sequence, were used to detect the total RNA extracted from 24-hpf embryos injected with pPG 10.0. This construct contained the upstream $10 \mathrm{~kb}$ and downstream $2.5 \mathrm{~kb}$ of zebrafish myf-5. If the myf-5 transcript was spliced correctly, an 841-bp PCR product was generated. If not, a 2.8 -kb PCR product was obtained. M: molecular marker, Bio 100; line 1: embryos were injected with linearized pPG10.0; line 2: untreated, wild-type embryos were the negative control.

plasmids (pP3.0 I 8 D1, -D2, -D3 and -D4) each with a deletion. The level of repression by each of the four constructs remained unchanged (Fig. 5). We generated plasmids pP3.0 I 8 D5 and -D6 by replacing fragment
$+660 /+815$ with an irrelevant 156-bp DNA fragment obtained from pGEM-T Easy vector. Surprisingly, in embryos injected with these plasmids, repression of GFP expression was completely restored (Fig. 5). Thus, we think

\section{Plasmids}

\section{Constructs}
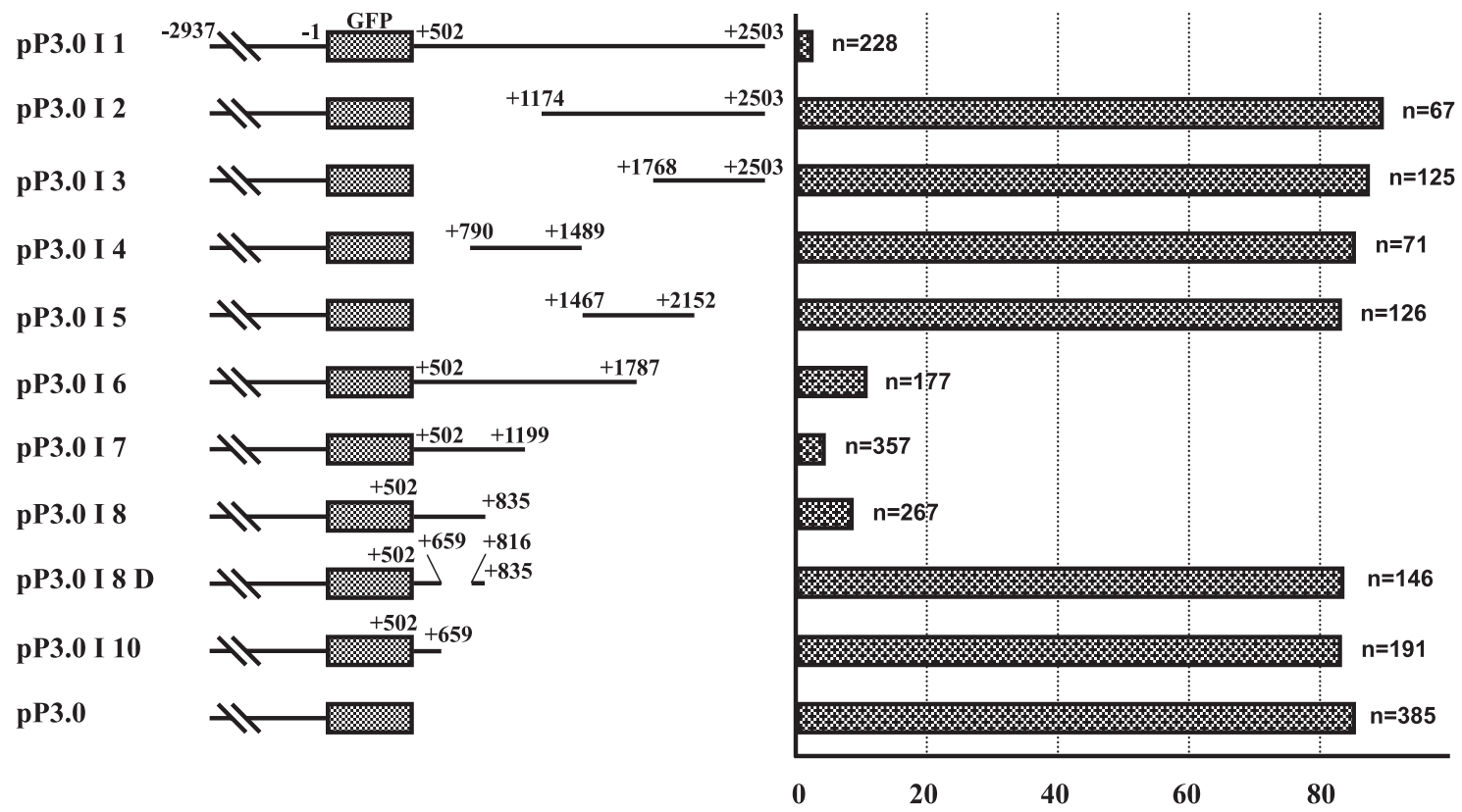

Somite Expression Rate (\%)

Fig. 3. The key repressive element for zebrafish $m y f-5$ is in intron 1. Deletion fragments from intron $1(+502 /+2503)$ were fused with the GFP gene and driven by the upstream regulatory region $(-2937 /-1)$ of zebrafish myf-5. $n$ : the total number of live embryos examined; somite expression rate: the number of embryos that expressed GFP in the somites divided by $n$. 
Plasmids

Constructs

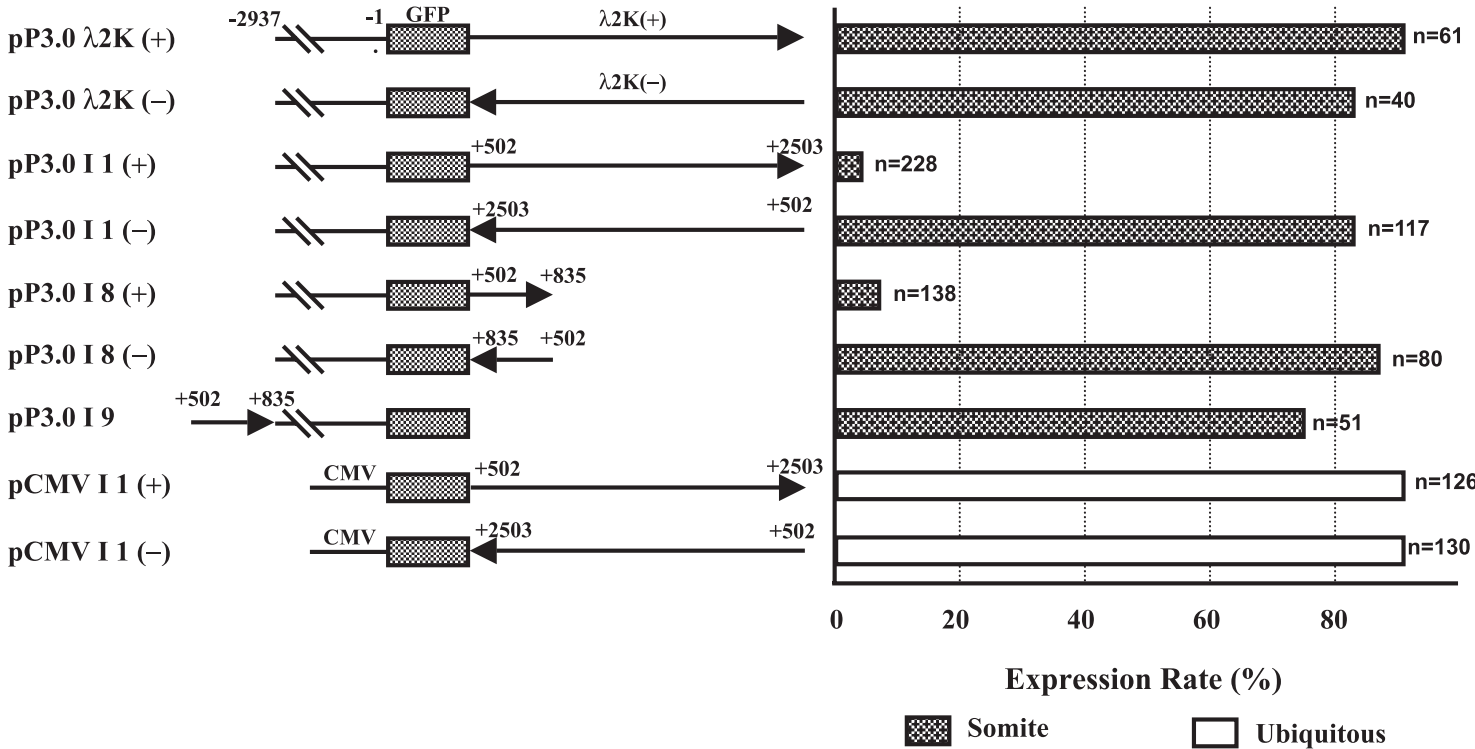

Fig. 4. The $m y f-5$ repressive element was orientation-, position- and promoter-specific. The reporter GFP gene driven by the regulatory sequence $-2937 /-1$ of zebrafish $m y f-5$ was engineered by two orientations of an irrelevant fragment $\lambda 2 \mathrm{~K}$ from $\lambda$ phage (the negative control), intron $1(+502 /+2503)$, or a key fragment $(+502 /+835)$ within intron 1. In plasmid pP3.0 I 9, the key fragment, $+502 /+835$, was constructed to locate in the upstream region of $-2937 /-1$. Two orientations of intron 1 driven by a nonspecific CMV promoter, pCMV I $1(+)$ and pCMV I $1(-)$, were constructed to express GFP and served as negative controls. $\lambda 2 \mathrm{~K}$ : a $2-\mathrm{kb}$ fragment from HindIII-cut $\lambda$ phage; $n$ : the total number of live embryos examined; somite expression rate: the number of embryos that expressed GFP in the somites divided by $n$; ubiquitous expression rate: the number of embryos that expressed GFP divided by $n$.

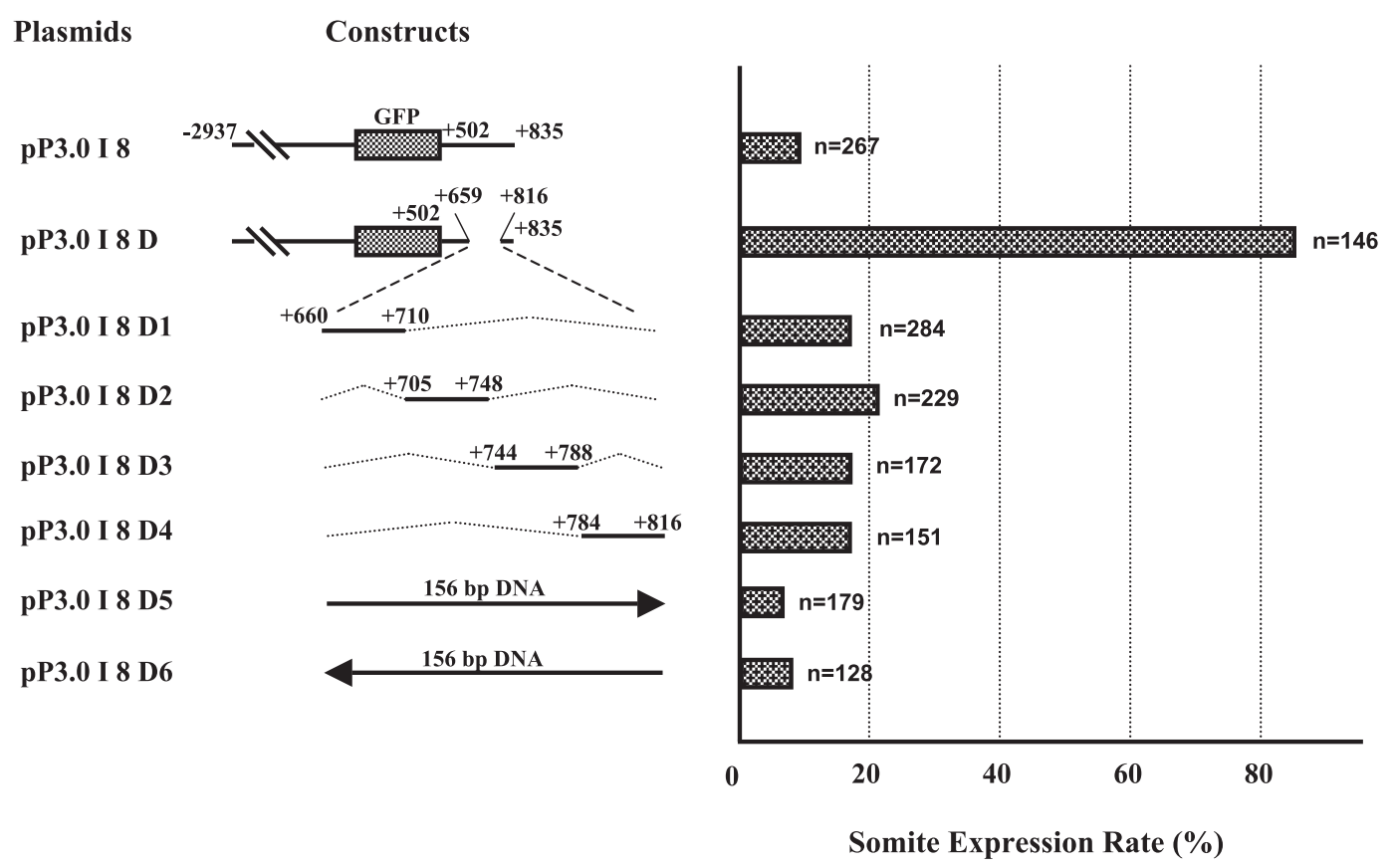

Fig. 5. A 156-bp spacer was essential for repression of myf-5 expression. pP3.0 I 8 was comprised of a regulatory fragment ( $-2937 /-1)$, GFP cDNA and the key element $(+502 /+835)$ of intron 1. Plasmid pP3.0 I 8 D was derived from pP3.0 I 8, but the region $+660 /+815$ was completely deleted. Plasmids pP3.0 I 8 D1, -D2, -D3 and -D4 represented different deletion clones within $+659 /+816$ fragment. An irrelevant, 156-bp fragment from pGEM-T easy vector was inserted in plasmids pP3.0 I 8 D5 and -D6. $n$ : the total number of live embryos examined; somite expression rate: the number of embryos that expressed GFP in the somites divided by $n$. 

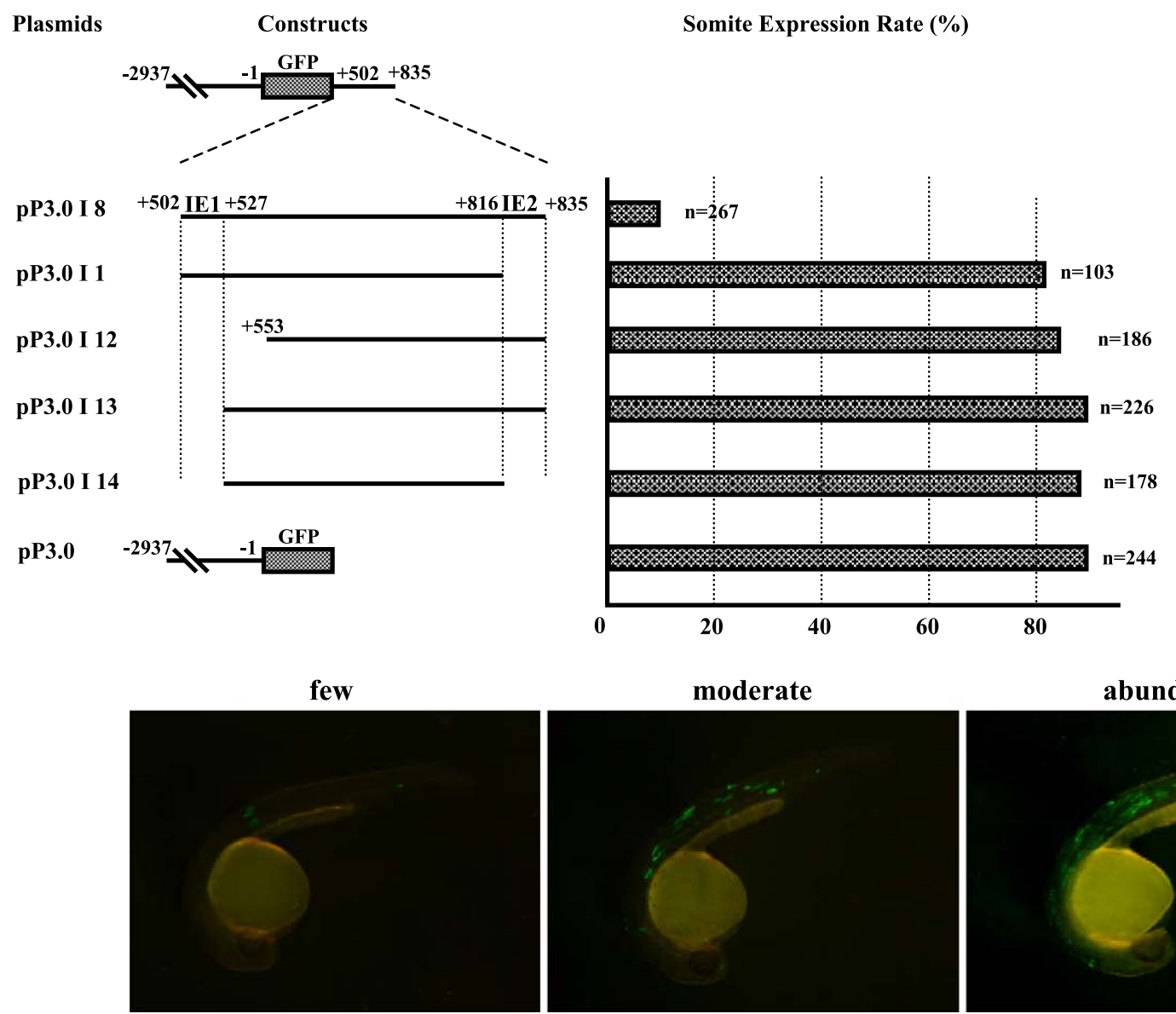

The percentages of 3 GFP-expressed levels (\%) $\square$ few $\square$ moderate $\square$ abundant

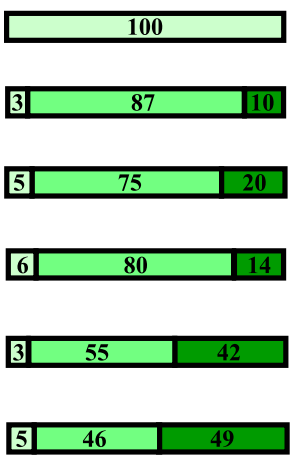

Fig. 6. Identification of the repressive elements, IE1 and IE2, within intron 1 of zebrafish $m y f-5$. To identify repressive elements, we constructed plasmids pP3.0 I 11, pP3.0 I 12, pP3.0 I 13 and pP3.0 I 14. The latter 3 plasmids had the myf-5 regulatory region $-2937 /-1$, GFP cDNA and the key element $(+502 /+835)$ of intron 1 except for IE1 (+502/+527). Plasmids pP3.0 I 11 and pP3.0 I 14 had the myf-5 regulatory region $-2937 /-1$, GFP cDNA and the key element $(+502 /+835)$ of intron 1 except for IE2 $(+817 /+835) . n$ : the total number of live embryos examined; somite expression rate: the number of embryos that expressed GFP in the somites divided by $n$. At $30 \mathrm{hpf}$, the level of GFP expression in GFP-positive embryos was classified based on the number of GFPpositive muscle fibers: few, 1-10; moderate, 11-40; and abundant, 41-70. The percentage of embryos exhibiting each level of expression was calculated by dividing the number of embryos at each level by the total number of GFP-positive embryos and multiplying by 100 .

that fragment $+660 /+815$ provides a spacer within the nucleosome that is essential for repression.

\subsection{Elements IE1 and IE2 within intron 1 are essential for repression}

In embryos microinjected with construct pP3.0 I 8, the rate of somite-specific expression was low and the number of muscle fibers with a GFP-positive signal was low. In contrast, in embryos injected with pP3.0 I 11, which lacked IE2 $(+816 /+835)$, the rate of somite-specific expression increased dramatically and the number of GFP-positive muscle fibers reached a moderate level (Fig. 6). Similar results were obtained from embryos injected with pP3.0 I 12 or pP3.0 I 13, both of which lacked IE1 $(+502 /+527)$ (Fig. 6). Furthermore, in the pP3.0- and pP 3.0 I 14-injected groups, the number of GFP-positive muscle fibers was abundant in about half the embryos. pP 3.0 I 14 lacked both IE1 and IE2, but the somite-specific expression rate was as high as it was in embryos injected with plasmids lacking either IE1 or IE2
(Fig. 6). Based on these results, IE1 and IE2 are essential for $m y f-5$ repression. We conclude that IE1 and IE2 work together, when 156 bp apart, to completely repress zebrafish myf-5.

\section{Discussion}

Known MRF, including chicken myf-5 (X73250), mouse myf-5 (AC021642), zebrafish myoD (AF318503) and myf-5 (Chen et al., 2001), tilapia (Oreochromis aruea) myoD (Chen et al., 2002), and fugu (Takifugu rubripes) $m y f-5$ and $m r f-4$ (AJ308546), are comprised of three exons and two introns. Intron 1 ranges from 0.3 to $2 \mathrm{~kb}$ and is longer than intron 2. In this study, we discovered a cisacting element in intron 1 that negatively affects zebrafish $m y f-5$ promoter activity. In human protooncogene c-myc, another bHLH factor, a cis-element (MIE1-3) in intron 1, functions as a silencer to block c-myc transcription (Bentley and Groudine, 1986).

In embryos injected with plasmids containing an upstream myf-5 region $(-9977 /-1,-8600 /-1,-2937 /$ 
-1 or $-290 /-1)$ fused with reporter GFP, the rate of somite-specific GFP expression was $85-96 \%$. When fragment $+502 /+835$ within intron 1 was linked downstream of each of the four constructs, the rate of somite-specific expression decreased to only $2-5 \%$ (Fig. 1). Thus, the novel repressive cis-element within intron 1 is in segment $+502 /+835$. The fragment from -9977 to -291 does not contain repressive elements. When the fragment containing $-9977 /-1$ fused with the intron is transferred into the embryos, the time when reporter GFP expression is observed in the somites corresponds to the time when endogenous myf-5 is expressed. This is shown by the whole mount in situ hybridization pattern (Fig. 1). This evidence suggests that cis-elements beyond $-10 \mathrm{~kb}$ may be bound by activators that are able to counteract this intron 1 silencer during the initiation stage of embryogenesis. Another possibility is that, during this early embryonic stage, the repressors that bind to intron 1 have yet to be translated.

Zebrafish myf-5 intron $1+502 /+835$ is an orientationand position-specific cis-element. Other such elements include the repressor of human Pax5 exon 1 (Rahman et al., 2001), the enhancer of the human norepinephrine transporter gene (Kim et al., 2001) and the enhancer of rat carnitine palmitoyltransferase $1 \beta$ first exon/intron boundary (Wang et al., 2002a). In zebrafish, IE1 (+502/ $+527)$ and IE2 $(+816 /+835)$ are the key motifs in intron $1(+502 /+835)$ that control repression. They are essential binding sites for repressors. IE1 and IE2 impede transcription only when they are located downstream of $m y f-5$ in the sense direction. If intron 1 is located upstream of $m y f-$ 5 , or in the anti-sense direction, there is almost no suppression of GFP activity. The repressor(s) that bind to IE1 and IE2 may form a static complex that prevents RNA polymerase from transcribing $m y f-5$ normally. A repressor that binds to the wrong location or strand will be less effective than a correctly bound repressor. The $m y f-5$ intron 1 repressive element does not suppress the activation of a CMV promoter. GFP expression remained unchanged in transgenic embryos injected with a fragment containing a CMV promoter, $m y f-5$ intron 1 and a GFP gene. Thus, the repressive element of intron 1 is myf-5-dependent.

We demonstrated that IE1 and IE2 repressive activity is strongly, spatially dependent. The silent or inhibitory effect of IE1 and IE2 can be mimicked by a construct with an irrelevant 156-bp DNA fragment inserted in pP3.0 I 8 D (Fig. 5, D5 and D6). However, when the number of nucleotides between IE1 and IE2 is less than $156 \mathrm{bp}$, repression is reduced greatly (Fig. 5). This suggests that the chromosomal structure around the intron 1 is a factor in zebrafish myf-5 repression. This is reminiscent of the Xenopus TFIIIA promoter, in which a nucleosome-dependent static loop activates the transcriptional machinery (Pfaff and Taylor, 1998). The repressive activity of intron 1 is dependent on the number of base pairs between motifs IE1 and IE2. We hypothesize that intron 1 may interact directly, via bound factors, with directional sites at IE1 and IE2 to form a complex that represses myf-5. Furthermore, proteins that bind to the distal upstream region of myf-5 may also interact with the proteins that bind to IE1 and IE2.

We previously reported that $-82 /-62$ is a cis-element that directs the somite-specific expression of zebrafish $m y f-5$ (Chen et al., 2003). We found a trans-acting factor that binds to the $-82 /-62$ region and functions as an activator (Lee et al., unpublished data). In this study, we found that a downstream region $(+502 /+835)$ within intron 1 is a cisacting element that represses $m y f-5$ expression. We hypothesize that a trans-acting factor binds to the $+502 /+835$ to form a repressor. The activator and silencer may be different binding proteins with different roles. However, it is possible that a trans-acting factor can function both as an activator and a repressor, depending on its binding sequence and/or its interaction with a specific co-factor. Transcription factors YY1 (Shrivastava and Calame, 1994; Shi et al., 1997) and NF-Y (Peng and Jahroudi, 2002) function in this way. Further research is needed to identify the activator and the silencer, their interaction and how trans-acting factors coordinate to regulate $m y f-5$ activation during myogenesis.

\section{Acknowledgements}

This work was supported by National Science Council, Republic of China, NSC92-2313-B002-078.

\section{References}

Bentley, D.L., Groudine, M., 1986. A block to elongation is largely responsible for decreased transcription of $c-m y c$ in differentiated HL60 cells. Nature 321, 702-706.

Buckingham, M., 1992. Making muscle in mammals. Trends Genet. 8, $144-149$

Carvajal, J.J., Cox, D., Summerbell, D., Rigby, P.W.J., 2001. A BAC transgenic analysis of the Mrf4/Myf5 locus reveals integrated elements that control activation and maintenance of gene expression during muscle development. Development 128, 1857-1868.

Chen, Y.H., Lee, W.C., Liu, C.F., Tsai, H.J., 2001. Molecular structure, dynamic expression, and promoter analysis of zebrafish (Danio rerio) $m y f-5$ gene. Genesis $29,22-35$.

Chen, Y.H., Liang, C.T., Tsai, H.J., 2002. Expression, purification and DNA-binding activity of tilapia muscle-specific transcription factor, MyoD, produced in Escherichia coli. Comp. Biochem. Physiol. 131B, 795-805.

Chen, Y.H., Lee, H.C., Liu, C.F., Lin, C.Y., Tsai, H.J., 2003. Novel regulatory sequence $-82 /-62$ functions as a key element to drive the somite-specificity of zebrafish myf-5. Dev. Dyn. 228, 41-50.

Cornelison, D.D.W., Wold, B.J., 1997. Single-cell analysis of regulatory gene expression in quiescent and activated mouse skeletal muscle sayllite cells. Dev. Biol. 191, 270-283.

Coutelle, O., Blagden, C.S., Hampson, R., Halai, C., Rigby, P.W., Hughes, S.M., 2001. Hedgehog signaling is required for maintenance of $m y f 5$ and $m y o \mathrm{D}$ expression and timely terminal differentiation in zebrafish adaxial myogenesis. Dev. Biol. 236, 136-150.

Dosch, R., Gawantka, V., Delius, H., Blumenstock, C., Niehrs, C., 1997. Bmp-4 acts as a morphogen in dorsoventral mesoderm patterning in Xenopus. Development 124, 2325-2334. 
Edmondson, D.G., Olson, E.N., 1993. Helix-loop-helix proteins as regulators of muscle-specific transcription. J. Biol. Chem. 268, 755-758.

Hacker, A., Guthrie, S., 1998. A distinct developmental programme for the cranial paraxial mesoderm in the chick embryo. Development 125 , $3461-3472$.

Hadchouel, J., Tajbakhsh, S., Primig, M., Chang, T.H.-T., Dubas, P., Rocancourt, D., Buckingham, M., 2000. Modular long-range regulation of Myf-5 reveals unexpected heterogeneity between skeletal muscles in the mouse embryo. Development 127, 4455-4467.

Hirsinger, E., Malapert, P., Dubrulle, J., Delfini, M.C., Duprez, D., Henrique, D., Ish-Horowicz, D., Pourquie, O., 2001. Notch signalling acts in ostmitotic avian myogenic cells to control MyoD activation. Development 128, 107-116.

Jones, C.M., Smith, J.C., 1998. Establishment of a BMP-4 morphogen gradient by $\mathrm{T}$ bug-range inhibition. Dev. Biol. 194, 12-17.

Kim, C.H., Ardayfio, P., Kim, K.S., 2001. An E-box motif residing in the Exon/Intron 1 junction regulates both transcriptional activation and splicing of the human norepinephrine transporter gene. J. Biol. Chem. 276, 24797-24805.

Kimmel, C.B., Ballard, W.W., Kimmel, S.R., Ullmann, B., Schilling, T.F., 1995. Stages of embryonic development of the zebrafish. Dev. Dyn. 203, 253-310.

Lin, G.F., Geng, X., Chen, Y., Qu, B., Wang, F., Hu, R., Ding, X., 2003. Tbox binding site mediates the dorsal activation of myf-5 in Xenopus gastrula embryos. Dev. Dyn. 226, 51-58.

Mei, W., Yang, J., Tao, Q., Geng, X., Rupp, R.A., Ding, X., 2001. An interferon regulatory factor-like binding element restricts Xmyf-5 expression in the posterior somites during Xenopus myogenesis. FEBS Lett. 505, 47-52.

Peng, Y., Jahroudi, N., 2002. The NFY transcription factor functions as a repressor and activator of the von Willebrand factor promoter. Blood 99, 2408-2417.

Pfaff, S.L., Taylor, W.L., 1998. Xenopus TFIIIA gene transcription is dependent on cis-element positioning and chromatin structure. Mol. Cell. Biol. 18, 3811-3818.
Rahman, M., Hirabayashi, Y., Ishii, T., Kodera, T., Watanabe, M., Takasawa, N., Sasaki, T., 2001. A repressor element in the $5^{\prime}$-untranslated region of human Pax 5 exon 1A. Gene 263, 59-66.

Rudnicki, M.A., Jaenisch, R., 1995. The MyoD family of transcription factors and skeletal myogenesis. BioEssays 17, 203-209.

Shi, Y., Lee, J.S., Galivin, K.M., 1997. Everything you have ever wanted to know about Yin Yang 1. Biophya Acta. 1332, F49-F66.

Shrivastava, A., Calame, K., 1994. An analysis of genes regulated by the multi-function transcriptional regulator Yin Yang-1. Nucleic Acids Res. 22, 5151-5155.

Smith, T.H., Kachinsky, A.M., Miller, J.B., 1994. Somite subdomains, muscle cell origins, and the four muscle regulatory factor proteins. J. Cell Biol. 127, 95-105.

Summerbell, D., Ashby, P.R., Coutelle, O., Cox, D., Yee, S.P., Rigby, P.W.J., 2000. The expression of Myf5 in the developing mouse embryo is controlled by discrete and dispersed enhancers specific for particular populations of skeletal muscle precursors. Development 127, 3745-3757.

Takahashi, S., Esumi, E., Nabeshima, V., Asashima, M., 1998. Regulation of the Xmyf-5 and XmyoD expression pattern during early Xenopus development. Zool. Sci. 15, 231-238.

Wang, G.L., Moore, M.L., Mcmillim, J.B., 2002a. A region in the first exon/intron of rat carnitine palmitoyltransferase $1 \beta$ is involved in enhancement of basal transcription. Biochem. J. 362, 609-618.

Wang, T.M., Chen, Y.H., Liu, C.F., Tsai, H.J., 2002b. Functional analysis of the proximal promoter regions of fish rhodopsin and myf-5 genes using transgenesis. Mar. Biotechnol. 4, 247-255.

Yablonka-Reuveni, Z., Rivera, A., 1994. Temporal expression of regulatory and structural muscle proteins during myogenesis of satellite cells on isolated adult rat fibers. Dev. Biol. 164, 588-603.

Yang, J., Mei, W., Otto, A., Xiao, L., Tao, Q., Geng, X., Rupp, R.A., Ding, X., 2002. Repression through a distal TCF-3 binding site restricts Xenopus myf-5 expression in gastrula mesoderm. Mech. Dev. 115, 79-89. 